\title{
Stability Analysis and Reconfiguration Strategy for Multi-agent $D$-formation Control
}

\author{
Alessandro Colotti ${ }^{1}$, Angelo Cenedese ${ }^{1}$, Sébastien Briot ${ }^{2}$, \\ Isabelle Fantoni ${ }^{2}$, and Alexandre Goldsztejn ${ }^{2}$ \\ 1 University of Padova, Department of Information Engineering, Padova, Italy \\ alessandro.colotti@studenti.unipd.it, angelo.cenedese@unipd.it \\ 2 CNRS, Laboratoire des Sciences du Numrique de Nantes, Nantes, France \\ \{Sebastien.Briot, Isabelle.Fantoni, Alexandre.Goldsztejn\}@ls2n.fr
}

\begin{abstract}
This paper introduces a new control approach to perform formation control tasks on multi-agent systems, called $D$-formation control. The $D$-formation controller is a gradient-descent control law that exploits a regularized potential function to efficiently achieve specific formations. Taking inspiration from the flocking of birds, this approach differentiates itself from the several formation control strategies that can be found in the literature thanks to its flexibility. In fact, the approach that is usually employed in formation control is to try to enforce a set of very strict constraints in order to achieve rigid, a priori defined structures. We will show that the $D$-formation approach greatly relaxes such conditions.

In this paper, the $D$-formation control problem is introduced, and the equilibrium configurations of the controller are characterized. Additionally, a strategy for switching from one stable equilibrium to another one, i.e. for changing the shape of the formation, is proposed.
\end{abstract}

Keywords: Formation control · Stability · Formation switching.

\section{Introduction}

In the last few years, the control of multi-agent systems has sparked a significant amount of interest in research [10], due to both their practical employment in various applications $[9,2]$ and the theoretical challenges arising in coordination and control of them $[12,11]$. A multi-agent system is a system formed by a network of mobile agents, usually aerial or terrestrial, which can interact and exchange information with each other in order to achieve a common goal. In particular, formation control [6], which is one of the most actively studied topics in the field, generally aims to drive a multi-agent system to achieve a desired geometrical pattern. Formation control has an impact on many real-world applications, like in autonomous Search and Rescue operations, environmental monitoring tasks, and ground and aerial coverage and surveillance. Several strategies to perform formation control can be found in the literature $[8,3]$, but the approach that is usually employed is to a priori define a rigid target structure, and to try to 
enforce a set of strict constraints in order to bring each agent to its pre-assigned position.

In this paper, it is presented a novel less-constrained approach, called $D$ formation control, that takes inspiration from the flocking of birds and exploits a virtual potential function to efficiently achieve specific formations. As it will be discussed, this approach removes the need to assign target positions beforehand, allowing each agent to naturally converge to a proper position. The objective of this work is to introduce, study and develop the $D$-formation control law. Specifically, the main contributions lie in the characterization of the equilibrium configurations of the $D$-formation control law and in the design of a generalized version of such controller, allowing for switching from one stable equilibrium to another one, that relies on the regularization paradigm.

\section{Multi-Agent Systems Modelling and Control}

\subsection{Dynamics of a Fleet of Agents}

An intuitive model for formation control is presented here. The context is that of a group of $N$ agents moving in a two or three-dimensional Euclidean space. A common choice is to model each agent as a single integrator [6], i.e.

$$
\dot{q}_{i}=u_{i}, \quad q_{i} \in \mathbb{R}^{f}
$$

where $q_{i}, i=1, \ldots, N$, represents the agent's position in 2D or 3D (respectively, $f=2,3)$, and $u_{i}$ represents the agent's input. These agents are said to be anonymous, in the sense that they all act alike and are identical. They are able to detect each other and, in particular, to measure their relative positions, but they have no knowledge about their absolute location.

\subsection{Control of Flocks, Gradient-Descent of a Virtual Potential Function}

As mentioned in the introduction, numerous strategies can be employed to control the behaviour of such group of agents. In this work, the focus is on a behavioural-based control approach based on gradient-descent.

Gradient-descent control relies on two key ingredients. Firstly, a virtual potential function $V(q)$ that represents the energy level of the whole $N$-agent flock in $\mathbb{R}^{f}$ is considered, where $q \in \mathbb{R}^{N f}$ denotes the state of the system. Secondly, the assumption is made that each agent is modeled as a point mass with fully actuated dynamics, as assumed in Eq. (1). In this way, it is possible to obtain a so-called gradient system of the form:

$$
\dot{q}=-\nabla V(q)
$$

Gradient systems, under shallow regularity conditions for $V(q)$, are guaranteed to converge to an equilibrium [4], and they can greatly simplify the study of the critical configurations of the agents. The stability analysis is reduced to the critical points' evaluation, and the subsequent spectrum analysis of the Hessian of a static potential function, as later discussed in Section 3. 


\subsection{Usual geometry of flocks: $\alpha$-lattices}

In this section, we detail a usual strategy able to provide a formal objective for geometry when controlling flocks, called $\alpha$-lattices [7].

For this strategy, a group of $N$ mobile agents is considered, and it is sought the set of configurations $q$ in which each agent $i$ is equally spaced with respect to all other agents belonging to its neighborhood $\mathcal{N}\left(q_{i}\right)$. In terms of inter-agent distances, this geometric objective can be attained by the solutions of the following set of constraints:

$$
\left\|q_{j}-q_{i}\right\|=D, \quad \forall j \in \mathcal{N}\left(q_{i}\right)
$$

The solutions of the set of constraints in (3) play the role of desired conformations of agents in a flock. The configurations that respect these constraints are called $\alpha$-lattices.

In the following section, we detail our new strategy for the control of flocks, called the $D$-formation problem.

\section{The $D$-formation Problem}

\subsection{Problem Formulation and Theoretical Analysis}

Differently from the $\alpha$-lattice formation problem, the $D$-formation problem can be stated as follows:

Problem 1. (D-formation) Find a distributed control law $u(\cdot)$ for a swarm of $N$ anonymous agents described by model (1) such that each single agent aims to be at the same distance $D$ from all the others.

From a geometric point of view, the target formation, which will be called $D$ formation, can be defined as follows:

$$
\left\|q_{j}-q_{i}\right\|=D, \quad \forall j \neq i
$$

It can be seen as a generalization of the $\alpha$-lattice, where each agent's neighbourhood contains the whole formation. However, with respect to the $\alpha$-lattice, here it is assumed that the information about agents position can be spread throughout the network (e.g. via multi-hop procedures or message broadcast) and the rationale behind this choice is to prevent the creation of clusters of agents and split formations, a risk that is inherently present in the objective stated by (3). Contrarily to $\alpha$-lattices, however, the $D$-formation configuration can be exactly achieved for agents' fleets up to $N=3$ or $N=4$ agents, considering the 2D or $3 \mathrm{D}$ case respectively. Therefore, in the remainder of the paper, $D$-formation configurations will indicate all equilibrium configurations that solve Problem 1 also in a soft sense (namely, not exactly).

In order to try to reach the $D$-formation, a virtual potential function $V(q)$ is defined with respect to a target inter-agent distance $D$ as:

$$
V(q)=\frac{1}{2} \sum_{i=1}^{N}\left[\frac{1}{N} \sum_{j \neq i}^{N}\left(d_{i j}-D\right)^{2}\right],
$$


where $d_{i j}=\left\|q_{j}-q_{i}\right\|$ is the distance between agents $i$ and $j$. The idea behind this virtual potential function is to embed the $D$-formation problem: having that $V(q)$ is non strictly convex, its minima are $D$-formation configurations. In particular, the configurations corresponding to $V(q)=0$ are related to the feasible exact ones, i.e. the solutions for which $d_{i j}=D$ for any agents.

Accordingly, the control law $u_{i}$ that makes the system a gradient system can be derived from (5), yielding:

$$
u_{i}=-\nabla_{i} V=\frac{2}{N} \sum_{j \neq i}^{N}\left(d_{i j}-D\right) \frac{q_{j}-q_{i}}{d_{i j}} .
$$

Clearly, although an exact $D$-configuration may not be physically achievable, thanks to the nature of the gradient control, the system will still converge to a point of local minimum, namely to a equilibrium configuration. Moreover, differently from the formation control approaches often found in the literature, where a target configuration is chosen and each agent is forced to go to its pre-assigned position, the $D$-formation control strategy simply relaxes an initial configuration to the local minima of the potential function $V(q)$.

Interestingly, the potential (5) can be decomposed into two terms as follows:

$$
V(q)=\underbrace{\frac{1}{2} \sum_{i=1}^{N}\left[\frac{1}{N} \sum_{j \neq i}^{N} d_{i j}\left(d_{i j}-D\right)\right]}_{V_{0}(q)} \underbrace{-\frac{1}{2} \sum_{i=1}^{N}\left[\frac{1}{N} \sum_{j \neq i}^{N} D\left(d_{i j}-D\right)\right]}_{V_{+}(q)},
$$

where $V_{0}(q)$ characterizes the equilibrium with a level of geometric symmetry $\left(V_{0}(q)=0\right)^{3}$ and $V_{+}(q)$ allows to discern among the different equilibrium configurations.

The analysis of the formations achievable by the proposed control law is performed, by exploiting the first order condition for the equilibria of the gradient system. More formally, the critical points of $V(q)$ can be found as the solutions of the unconstrained optimization problem:

$$
q^{*}=\underset{q \in \mathbb{R}^{N f}}{\arg \min } V(q) \Longrightarrow q^{*}: \nabla V\left(q^{*}\right)=0
$$

where $\left\{q^{*} \in \mathbb{R}^{N f}\right\}$ represents the set of equilibrium configurations.

\subsection{Numerical Analysis for Equilibria Evaluation}

Given the complexity of the considered potential function, finding the equilibrium configurations $q^{*}$ is performed using numerical tools.

By employing the IBEX library [1], which is a $\mathrm{C}++$ library for constraint processing over real numbers, the solutions of the system of equations given

\footnotetext{
${ }^{3}$ To attain $V_{0}(q)=0$, the agent distances tend to balance among themselves between
} the set with $d_{i j}>D$ and that with $d_{i j}<D$. 
by (8) are sought and all the $D$-formation planar equilibria have been evaluated for fleets of up to 5 agents, and their stability has been assessed studying the spectrum of the Hessian of the potential function. Unfortunately, though, this approach is computationally costly and only small planar systems can be solved.

Hence, in order to have a wider perspective on the controller's capabilities, a second approach to evaluate local minima has been developed. This method is implemented as a Multi-Start procedure that exploits the trust-region algorithm [5], an iterative algorithm that evaluates a local minimum starting from a given initial configuration. The agents' initial positions are picked uniformly over a $100 \times 100 \mathrm{~m}$ surface in the planar case, or a $100 \times 100 \times 100 \mathrm{~m}$ cube in the $3 \mathrm{D}$ one. Obviously, we cannot guarantee that this approach is able to find all local minima, but it was able to identify several ones for the tested formations.

These results are summarized with some examples in Tab. 1 and Tab. 2, showing also the values of the potential function. For all the simulations, a value $D=5 m$ has been chosen.

\begin{tabular}{|c|c|c|c|c|c|c|c|}
\hline$N$ & \multicolumn{2}{|c|}{3} & \multicolumn{3}{|c|}{4} & \multicolumn{2}{|c|}{10} \\
\hline & $\bullet \bullet \bullet$ & $\begin{array}{ll} & \bullet \\
\bullet & \bullet\end{array}$ & 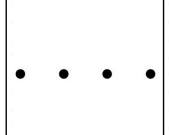 & $\begin{array}{lll} & \bullet & \\
\bullet & \bullet & \bullet\end{array}$ & $\begin{array}{ll}\bullet & \bullet \\
\bullet & \bullet\end{array}$ & $\begin{array}{ccc} & \bullet & \bullet \\
\bullet & & \bullet \\
\bullet & \bullet & \\
& \bullet & \bullet\end{array}$ & $\begin{array}{lll}\bullet & \bullet & \bullet \\
\bullet & \bullet & \bullet \\
\bullet & \bullet & \bullet\end{array}$ \\
\hline$\overline{V_{0}}$ & 0 & 0 & 0 & 0 & 0 & 0 & 0 \\
\hline$V_{+}$ & 2.78 & 0 & 6.25 & 2.51 & 1.07 & 12.49 & 12.36 \\
\hline$\overline{\bar{V}}$ & 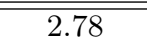 & 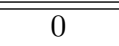 & 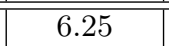 & 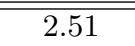 & $\bar{~} 1.07$ & "12.49 & $\bar{~} 12.36$ \\
\hline Stable & $\mathrm{No}$ & Yes & No & No & Yes & Yes & Yes \\
\hline
\end{tabular}

Table 1. Examples of $D$-formation equilibria in 2D. Full set of equilibria for $N=3,4$; pair of stable equilibria for $N=10$.

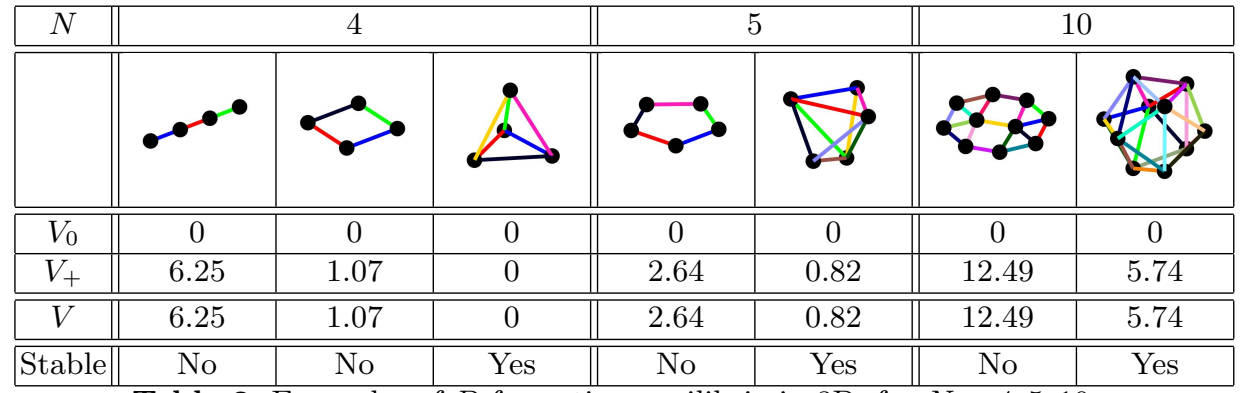

Table 2. Examples of $D$-formation equilibria in 3D, for $N=4,5,10$. 


\section{$4 \quad D$-formation Switching Control}

\subsection{Switching Formation Task}

The control law (6) allows to move from a generic initial displacement of the agents towards one of the $D$-formation equilibria (local minima of $V(q)$ ), so as to solve Problem 1. It is now to understand whether it is possible to switch among from one local minima to another one and converge to a specific chosen configuration, as usually done in formation control. Formally, the following problem can be stated as

Problem 2. (Switching formation) Find a distributed control law $u(\cdot)$ for a swarm of $N$ anonymous agents to switch between $D$-formations.

whose solution objective is to switch between different stable configurations. To this aim, a regularized version of the potential (5) is employed, since it allows to favour particular agents' arrangements exploiting a priori information on the equilibrium configurations. In practice, the idea is to add a regularization term $R(q)$ to the potential function $V(q)$, in order to penalize the initial unwanted equilibrium, which becomes a repulsive configuration. Thanks to $R(q)$, the action of the controller forces the agents potential $V(q)$ outside the basin of attraction of the initial equilibrium, to allow the convergence towards other configurations.

\subsection{Fixed Target Controller}

In this context, it is chosen to design a control law that drives the configuration towards a specific equilibrium $\widetilde{q^{*}}, \widetilde{q^{*}} \in\left\{q^{*}\right\}$ that is assumed to be known. In order to achieve this task, the idea is to consider the following potential:

$$
V_{c}(q)=V(q)+R(q) \quad \text { with } \quad R(q)=\frac{1}{2} k_{c}\left\|\tilde{q^{*}}-q\right\|^{2}, \quad k_{c} \in \mathbb{R}_{+}
$$

Clearly, $R(q)$ is a convex function with unique minimum represented by $\widetilde{q^{*}}$. Since a gradient-descent controller is employed, the only action of the regularization term is to push the formation towards the configuration $\widetilde{q^{*}}$, in particular when the current configuration is distant from the desired $\widetilde{q}^{*}$. Being it an equilibrium configuration for $V(q), \widetilde{q^{*}}$ is also an equilibrium for the whole potential $V_{c}(q)$.

The agents' control law is then computed as follows:

$$
u_{i}=-\nabla_{i} V_{c}(q)=\frac{2}{N}\left[\sum_{j \neq i}^{N}\left(d_{i j}-D\right) \frac{q_{j}-q_{i}}{d_{i j}}\right]+k_{c}\left(\widetilde{q_{i}^{*}}-q_{i}\right) .
$$

At the beginning of the control action, when the formation is near $q^{*} \neq \widetilde{q^{*}}$, the effect of $V(q)$ is negligible and each agent is driven by $-\nabla_{i} R(q)$ towards $\widetilde{q_{i}^{*}}$. As the formation leaves the initial basin of attraction of the initial equilibrium, the fleet overcomes a relative maximum of $V(q)$, to finally descend to the $\widetilde{q^{*}}$. It is worthwhile to note that an accurate tuning of the parameter $k_{c}$ is required, 


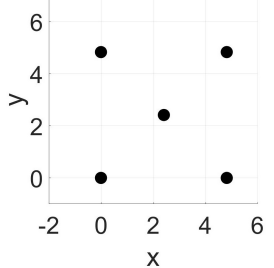

(a)

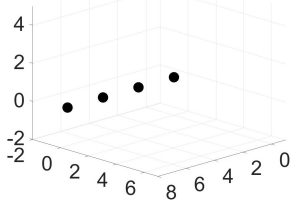

(e)

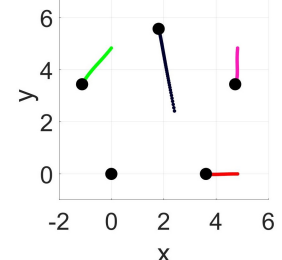

(b)

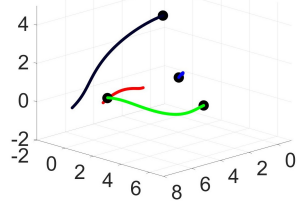

(f)

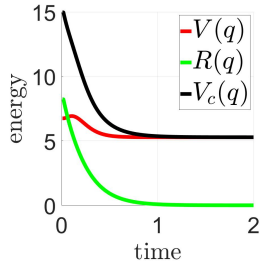

(c)

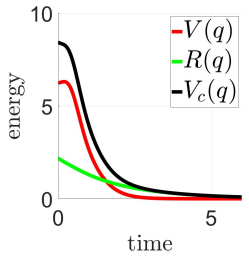

(g)

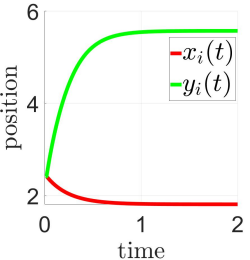

(d)

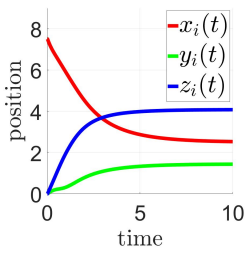

(h)

Fig. 1. Fixed target equilibrium controller. (a)-(d): 2D case with $N=5$ and (e)-(h): $3 \mathrm{D}$ case with $N=4$. Initial (a),(e) and final (b),(f) configurations; (c)-(g): evolution of the potential energy terms; (d)-(h): evolution of the coordinates of a single agent.

in order to guarantee the switching between the attraction basins of the two equilibria.

Figure 1 reports two examples of switching configuration task, related to a $2 \mathrm{D}$ case (switch between soft $D$-formations) and to a $3 \mathrm{D}$ case (switch towards an exact $D$-formation). The plots of the energy potentials (Fig. $1(\mathrm{c}),(\mathrm{g}))$ allow to remark that local minima are present in $V(q)$ (red curves) and the role of the regularization term $R(q)$ in moving from the initial equilibria. Also, the final value of $V_{c}(q)$, equal to that of $V(q)$, is zero only in the case of exact $D$-formation (Fig. 1(g)).

\section{Conclusions}

In this work an approach to formation control has been proposed, based on the definition of a suitable potential energy function, which aims at solving the $D$ formation problem where all agents of a group aim at being at the same distance $D$ from each other. This problem turns out to be a non-convex optimization problem, where the only exact solution reachable is the triangular configuration in $2 \mathrm{D}$ and the tetrahedron configuration in $3 \mathrm{D}$, while multiple minima are solving the problem in a soft sense for generic number of agents in 2D-3D.

The proposed solution to this problem exploits to the theory of gradient systems and allows the design of a distributed control law that drives the multiagent system to one equilibrium point within the set of available configurations; furthermore, this law can be adapted in order to reach a specific desired configuration through a switching equilibria control. 
However, from a practical point of view, it would be better to be able to privilege a set of equilibria, characterized by properties in common, more than a specific equilibrium configuration (chosen $a$ priori). This aspect, together with a strategy to automatically tune the gain of the switching controller is the subject of future study. Another possible line of research regards the in-depth study of the stability of the configurations: since the potential function is not convex, the presence of isolated minima with different values of $V(q)$ has been shown but the analysis of these values alone is not sufficient to draw conclusions on the attraction basins, while this issue becomes important to optimize the switching formation control effort.

\section{References}

1. Ibex library website, http://www.ibex-lib.org/

2. Alexis, K., Nikolakopoulos, G., Tzes, A., Dritsas, L.: Coordination of helicopter uavs for aerial forest-fire surveillance. In: Applications of intelligent control to engineering systems, pp. 169-193. Springer (2009)

3. Anderson, B.D.O., Yu, C., Fidan, B., Hendrickx, J.M.: Rigid graph control architectures for autonomous formations. IEEE Control Systems Magazine 28(6), 48-63 (2008)

4. Hirsch, M.W., Devaney, R.L., Smale, S.: Differential equations, dynamical systems, and linear algebra, vol. 60. Academic press (1974)

5. Moré, J., Sorensen, D.: Computing a trust region step. SIAM Journal on Scientific and Statistical Computing 3, 553-572 (1983)

6. Oh, K.K., Park, M.C., Ahn, H.S.: A survey of multi-agent formation control. Automatica 53, 424-440 (2015)

7. Olfati-Saber, R.: Flocking for multi-agent dynamic systems: Algorithms and theory. IEEE Transactions on Automatic Control 51(3), 401-420 (2006). https://doi.org/https://doi.org/10.1109/TAC.2005.864190

8. Park, M.C., Ahn, H.S.: Distance-based acyclic minimally persistent formations with non-steepest descent control. International Journal of Control, Automation and Systems 14(1), 163-173 (2016)

9. Prokaj, J., Medioni, G.: Persistent tracking for wide area aerial surveillance. In: The IEEE Conference on Computer Vision and Pattern Recognition (CVPR) (June 2014)

10. Shamma, J.: Cooperative control of distributed multi-agent systems. John Wiley \& Sons (2008)

11. Yang, H., Han, Q., Ge, X., Ding, L., Xu, Y., Jiang, B., Zhou, D.: Fault-tolerant cooperative control of multiagent systems: A survey of trends and methodologies. IEEE Transactions on Industrial Informatics 16(1), 4-17 (2020)

12. Zhao, S., Dimarogonas, D.V., Sun, Z., Bauso, D.: A general approach to coordination control of mobile agents with motion constraints. IEEE Transactions on Automatic Control 63(5), 1509-1516 (2018) 